\title{
Il problem-based learning come metodologia di aggiornamento degli infermieri: esperienza nelle realtà dei gruppi ristretti di una residenza protetta per anziani Problem-based learning as a training methodology for nurses: experience in small groups in a retirement home
}

\author{
Sara Mattioli \\ Studio Infermieristico MeC, Sassuolo (MO), Italia
}

\section{RIASSUNTO}

L'adulto è visto come un essere che continua ad apprendere. Il modo in cui esso apprende è descritto nell'andragogia, di cui il massimo esponente è Malcom Knowles. Nel modello andragogico risulta centrale il ruolo che riveste l'esperienza dell'adulto nel modo di apprendere e di questo contesto fanno parte gli esercizi del problem solving. Negli anni '60, il neurologo Barrows inizia a introdurre la metodologia del problem-based learning nelle sue lezioni, proponendo una metodologia di apprendimento basata sulla risoluzione di un problema, reale o realistico, da parte dei discenti che, collaborando tra loro, cercano di dargli una soluzione, in modo che le nozioni apprese possano essere applicate alla realtà lavorativa quotidiana. Questa metodologia è stata applicata a un piccolo gruppo di dieci infermieri di una residenza protetta per anziani, riguardo alla cura delle lesioni che maggiormente si trovano a trattare nella loro realtà, al fine di vedere concretamente se potesse essere uno strumento valido da proporre come corso di aggiornamento, in alternativa agli attuali corsi in plenaria. È stato somministrato loro un questionario di 10 domande, a risposta multipla, relativo alla pratica delle medicazioni di tre tipologie di lesioni: lesioni da pressione, skin tears e lesioni venose. Questo è stato somministrato all'inizio e al termine del lavoro di gruppo, svolto su tre casi reali di lesioni e sono stati messi a confronto i risultati. I risultati ottenuti sono stati soddisfacenti, gli infermieri hanno dimostrato di avere imparato nuove nozioni e corretto quelle errate: si passa da un $61 \%$ di risposte corrette nel test iniziale a un $97 \%$ in quello finale. Il lavoro è stato apprezzato anche dagli stessi infermieri che lo hanno svolto, si sono sentiti coinvolti e hanno partecipato attivamente e con attenzione al corso. Questi aspetti positivi suggeriscono che si potrebbe adottare maggiormente questa metodologia applicandola ai corsi di diverse figure professionali (infermieri, medici, OSS, ecc.)

\section{ABSTRACT}

The adult is seen as being who continues to learn. The way in which it learns is described in andragogy, whose leading exponent is Malcom Knowles. The role played by the adult's experience in the way of learning is central in the androgical model and problemsolving exercises are part of this context. In the 1960's the neurologist Barrows begins to introduce the methodology of problem-based learning in his lessons, by proposing a learning methodology based on the resolution of an issue, real or realistic, by learners. Learners had to cooperate with each other trying to find a solution and they would have applied those learned lessons to their working daily reality. This methodology was applied to a small group of ten nurses, who were working in a protected residence for the elderly, in order to verify, in a very concrete way, whether it could be considered as a valid instrument and proposed in the form of an updating course, as a possible alternative to the current plenary courses. The nurses were asked to answer a questionnaire based on ten multiple choice questions, concerning the practice of medication to three types of ulcers: pression ulcers, skin tears and venous ulcers. The questionnaire was administered at the beginning and end of group work carried out on three real cases of ulcers and the results were compared. The results obtained were satisfactory, nurses shown to have learned new knowledge and correct those incorrect: they have increased from $61 \%$ of correct answers in the initial test at the $97 \%$ by the end. The work was also appreciated by the nurses themselves because they felt involved and participated actively and carefully to the course. These positive aspects suggest that you could adopt this methodology by applying it to the various professional figures (nurses, doctors, socio healthcare workers, etc.). 


\section{INTRODUZIONE}

Il tema dell'apprendimento degli adulti è studiato fin dall'antichità. Molti dei grandi maestri filosofi hanno scritto a riguardo e, dopo tanti studi, si è arrivati a capire che l'adulto è un soggetto in apprendimento e come tale, capace di accrescere le proprie conoscenze. La differenza che si nota nel confrontare il discente bambino con quello adulto è che, mentre il primo si deve ancora formare, l'adulto racchiude in sé esperienze passate che lo caratterizzano, ha una vita lavorativa, ha degli interessi, ha una propria identità. Questo lo porta a non riuscire a memorizzare tutto ciò che gli viene insegnato, ma a fissare solo quei concetti a lui utili. Possono essere concetti utili a interessi personali o lavorativi ma, per apprenderli, l'adulto deve poterli inserire in suo contesto quotidiano. Solo trovando un riscontro nella propria vita egli potrà accrescere le conoscenze, mentre tutto quello che non riuscirà ad applicare tenderà nel tempo a essere dimenticato. Questa potrebbe essere la chiave giusta da applicare ai corsi di aggiornamento per le figure professionali: fornire nozioni nuove o aggiornate che possono essere applicate già dal momento in cui finisce il corso alla propria realtà lavorativa.

Il problem-based learning (PBL) è uno degli strumenti forniti dall'andragogia ${ }^{1}$ che può essere introdotto a supporto dell'apprendimento degli adulti. Lo scopo di questo studio è sperimentare il PBL in un contesto reale affinché possa diventare una proposta innovativa per i corsi di aggiornamento di piccoli gruppi di professionisti e dimostrare che possa essere un valido strumento attraverso il quale colmare nell'immediato lacune, correggere errori, implementare le conoscenze che poi rimarranno più a lungo e che potranno essere messe subito in pratica nella clinica quotidiana.

L'acronimo PBL significa problem-based learning cioè un apprendimento basato sui problemi.

Nello specifico questo metodo vuole essere un modo di apprendere basato su problemi reali di cui i discenti devono trovare la soluzione. ${ }^{2,3}$ E' una tecnica pedagogica caratterizzata da forte interattività e basata sul coinvolgimento attivo dei discenti, L'apprendimento non si attua ascoltando, bensì facendo, ricercando autonomamente le soluzioni al puzzle proposto. Gli esercizi di PBL consentono, quindi, un collegamento tra l'apprendimento teorico-cognitivo (knowing that) e quello tecnico-operativo (knowing how), arricchendo notevolmente l'efficacia della lezione.

La novità che sicuramente questo metodo di apprendimento propone è il ruolo che assume l'alunno (o il discente). Questo non è più partecipante passivo di una lezione frontale ma diventa protagonista della lezione. Questo non solo risulta essere un vantaggio per ciò che vanno ad apprendere ma fa si che ci sia una crescita personale di relazione con gli altri in quanto questa metodologia li mette di fronte al confronto costruttivo , ascoltando attivamente e confrontandosi con gli altri. Vengono quindi messe in gioco le competenze professionali e le competenze comunicative e sociali.

I gruppi devono essere formati da un minimo di 8 a un massimo di 12 persone. Durante la fase iniziale viene indicato un moderatore e un segretario.

Schmidt ${ }^{4}$ individua sette fasi (seven steps) di lavoro del PBL:

Step 1: Chiarire termini e concetti non completamenti comprensibili.

Step 2: Definire il problema.

Step 3: Analizzare il problema.

Step 4: Formulare un inventario sistematico (ossia schematizzare) delle spiegazioni desunte dallo step 3.

Step 5: Formulare obiettivi di apprendimento.

Step 6: Raccogliere informazioni aggiuntive al di fuori del gruppo.

Step 7: Sintetizzare e valutare le informazioni acquisite di recente. 4

Il tutor, dopo avere presentato il problema, guida i discenti attraverso questi sette steps.

Lotti ${ }^{5}$ nel 2005 aggiunge altri 3 steps:

Step 8: Formulare domande di ricerca.

Step 9: Valutare il lavoro di gruppo.

Step 10: Valutare il lavoro personale.

Il tutor in questo caso non è solo un insegnante che trasmette nozioni, ma diventa un facilitatore di apprendimento e trasmettitore di conoscenza. Pianifica i moduli didattici fissando gli obiettivi da raggiungere e i punti da toccare necessariamente durante la lezione. Il ruolo del tutor è attivo nel gruppo, promuove discussioni e confronti e interviene se si divaga troppo dal tema principale.

Uno degli studi più recenti riguardo eziologia e prevalenza delle lesioni pone ai primi tre posti le seguenti tipologie (con relative percentuali di riscontro): Ulcere da pressione $28,1 \%$, Ulcera venosa $21,7 \%$, Ulcera post traumatica $12,1 \% .6$ Secondo gli studi questi numeri sono destinati a salire e sia negli ospedali che nelle case di riposo per anziani i numeri sono tra i più alti. Per questo è necessario che gli operatori, oltre che a compiere un accurato lavoro sulla prevenzione, si dedichino anche ad aggiornamenti continui volti a migliorare la cura delle lesioni. In questo contesto il PBL potrebbe essere lo strumento meglio applicabile per far diventare l'apprendimento attivo e immediatamente applicabile per contenere questi numeri. Diversi studi, anche nell'ambito dell'educazione continua in medicina e della formazione a distanza, sono stati condotti sulla metodologia del PBL dall'Istituto Superiore di Sanità con risultati notevolmente positivi di superamento delle prove finali. ${ }^{6}$

\section{MATERIALI E METODI}

Date le premesse, a supporto della mia ipotesi e per poter applicare il metodo del PBL e verificarne la validità 
in termini di risultati, mi sono rivolta alla casa protetta "Il pellicano" di Bazzano in provincia di Bologna, dove ho prestato servizio come infermiera qualche anno fa. Questa è una piccola realtà che mostrava le caratteristiche idonee per la mia ricerca: un numero ristretto di infermieri (10), alcune casistiche di lesioni che non sono riusciti a medicare, alcune lacune da colmare in termini di conoscenza riguardo alla medicazione delle lesioni.

Sono infermieri che hanno un'età compresa tra i $37 \mathrm{e}$ i 60 anni, lavorano tutti da più di 15 anni con questa mansione, e da più di 10 nella realtà delle case di riposo.

Mi sono rivolta alla direttrice della struttura spiegandole la mia idea che è stata ben accolta, dandomi quindi il consenso di lavorare con gli infermieri.

Abbiamo stabilito le giornate di lavoro e deciso di dividere in due gruppi gli infermieri per motivi lavorativi relativi ai loro turni.

Gli incontri sono stati svolti nei mesi di giugno e settembre (mesi in cui non vi erano infermieri in ferie). Le ore messe a disposizione sono state due per ogni incontro, tempistiche non previste dalle caratteristiche che dovrebbe avere un incontro di PBL ma è stato adattato il corso in base alle loro esigenze. Anche la parte dello studio a casa si è mostrata una criticità, che potrebbe essere considerata anche in una possibile applicazione della metodologia nei corsi di aggiornamento, per cui abbiamo dovuto svolgerla in sede di lavoro.

Il materiale che ho utilizzato è stato un questionario di dieci domande a risposta multipla per capire il livello di conoscenza di alcuni contenuti sulle ulcere; tre casi reali di ulcere: una lesione da pressione, una skin tears e un'ulcera venosa che ho seguito, delle quali sono state trasmesse da proiettore le immagini; un fascicolo dove erano riassunte le caratteristiche principali e i trattamenti da eseguire e non, relativi a queste tre tipologie di lesioni (Figure 1-3).

Sono stati quindi applicati i passi previsti dalla metodologia del PBL.

È iniziato il lavoro di gruppo spiegando la metodologia che avremmo utilizzato in quanto nessuno di loro la conosceva. È stato poi suddiviso il tempo che avevamo a disposizione e ho iniziato a somministrare il questionario che ha impiegato pochi minuti.

Dopo siamo passati alla proiezione dei tre casi e una piccola descrizione di anamnesi dei pazienti. Di queste dovevano individuare il problema (che nel nostro caso specifico riguardava il trattamento delle lesioni in esame) e quindi riferire che tipo di lesioni erano e le loro caratteristiche (sede, bordi, segno prevalente). C'è stata quindi la discussione del gruppo per raggiungere gli obiettivi e li abbiamo scritti sotto a ognuno delle foto. Ė stato poi svolto il lavoro di studio individuale e quindi della risoluzione del problema tramite il materiale che avevo fornito loro.

Gli infermieri hanno illustrato le loro risposte e io ho fornito loro le linee guida regionali sulla cura di queste spe-

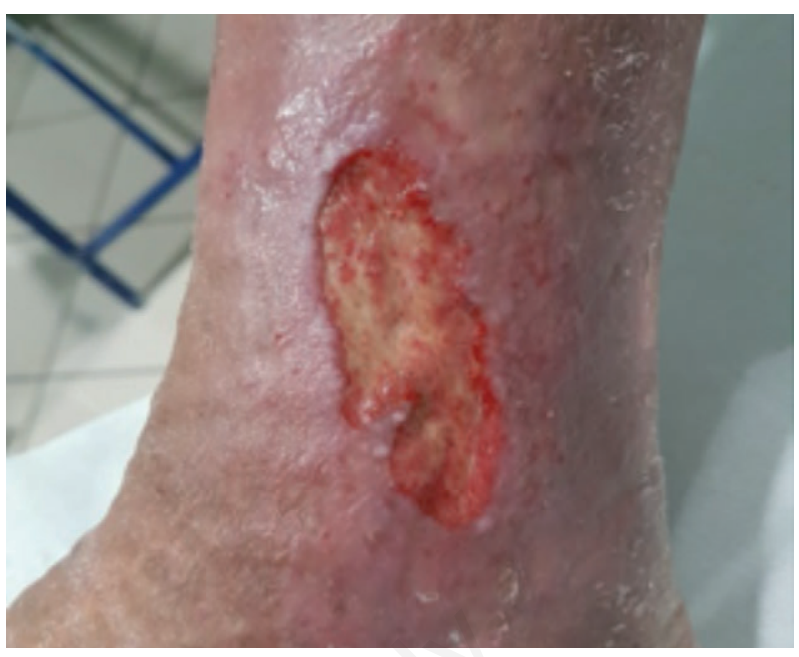

Figura 1. Lesione venosa.

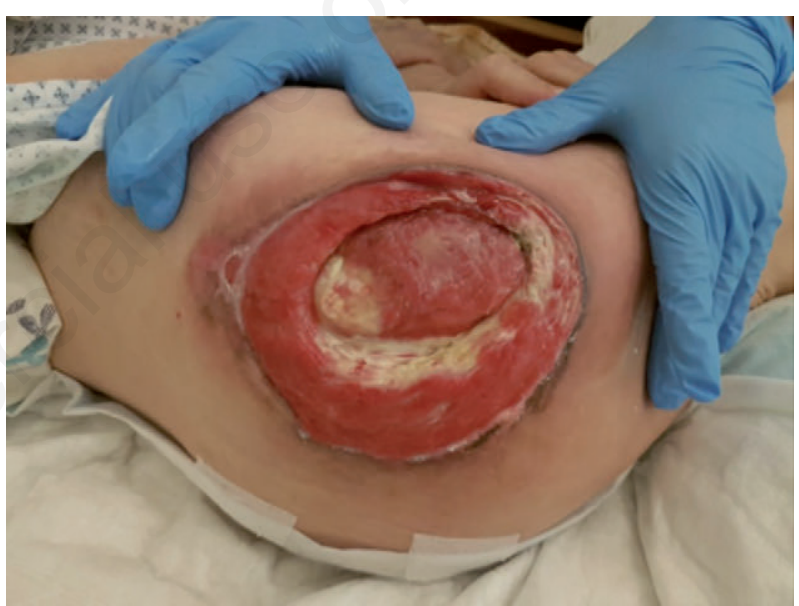

Figura 2. Lesione da pressione.

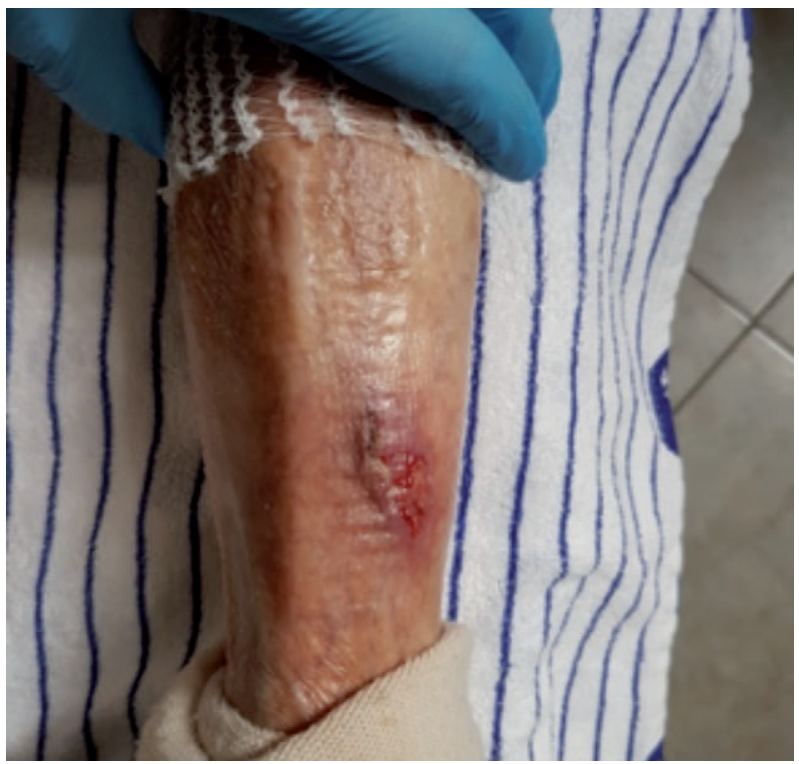

Figura 3. Skin tears. 
cifiche lesioni e i risultati raggiunti sino quel momento. Ho risposto alle loro domande e dubbi a riguardo di ciò che era stato fatto e ho poi ripetuto alla fine lo stesso questionario per vedere se le risposte sbagliate sarebbero state corrette e capire quindi se l'intervento fosse stato positivo a livello sia di conoscenza sia di applicabilità del metodo.

\section{RISULTATI}

Domanda 1. Segna tra i seguenti il prodotto corretto riguardo la DETERSIONE di un' ulcera:

Acqua e sapone

$(30 \%)$

Fisiologica o ringer lattato

Acqua del rubinetto

$(70 \%)$ Risposta corretta

Acqua minerale - naturale

$(0 \%)$

Domanda 2. Segna la tempistica appropriata per i cambi di medicazione:

Tutti i giorni

Dipende dall'essudato

(60\%) Risposta corretta

Dipende dai giorni relativi al

cambio indicati dalla casa

costruttrice della medicazione $(20 \%)$

Relativamente alle tempistiche

di reparto

Domanda 3. Ogni quanto va eseguita la valutazione di una lesione?

Tutti i giorni

Ad ogni cambio di

medicazione

$(100 \%)$ Risposta corretta

Secondo le necessità di reparto $(0 \%)$

Una volta al mese

Domanda 4. Segna la definizione corretta di skin tears:

Lesione dell'anziano e

dell'adulto di origine traumatica

che interessa gli strati superficiali

della cute

Lesione traumatica che si

verifica sulle estremità degli

anziani, risultato di forze di

taglio o sfregamento, le quali

separano l'epidermide

dal derma

(70\%) Risposta corretta

Lesione data dallo sfregamento

della cute su una superficie

piana che fa separare la cute

a diverse profondità

Lesione traumatico che si

verifica sulle estremità degli

anziani, risultato di forze di taglio o

sfregamento, le quali separano

il derma dall'ipoderma
Domanda 5. Nella gestione delle skin tears quale tra i seguenti trattamenti è il più appropriato da applicare alle pelle rimasta attaccata:

Sollevarla ma non staccarla

definitivamente permettendone

poi il distacco naturale

Tagliare con forbici la pelle

ritirata

Conservare la pelle ritirata

stendendola sulla lesione e

facendola aderire con

steril-strip

Raggruppare la pelle rimasta

verso il sito di attaccatura e

ripiegarla su se stessa

Domanda 6. Nella gestione delle lesioni venose quale tra i seguenti trattamenti si definisce il gold standard:

Bendaggio compressivo con

medicazione appropriata $\quad(30 \%)$ Risposta corretta

Scarico dell'arto interessato $\quad(70 \%)$

Medicazioni avanzate $\quad(0 \%)$

Rivascolarizzazione $\quad(0 \%)$

Domanda 7. Qual è l'intervento più importante per la guarigione delle lesioni da pressione?

Scarico del peso

$(0 \%)$ Risposta corretta

Medicazione giusta

$(100 \%)$

Apporto proteico

Fibrinolisi tempestiva

$(0 \%)$

Domanda 8. Tra le tecniche di debridement più selettive e indolori sono incluse:

Sbrigliamento autolitico e

enzimatico

Sbrigliamento meccanico

Sbrigliamento chirurgico

(70\%) Risposta corretta

Nessuna delle precedenti

$(30 \%)$

$(0 \%)$

$(0 \%)$

Domanda 9. Quale tra queste è il principale prodotto per la disinfezione di un'ulcera?
Acqua ossigenata
$(0 \%)$
Iodio povidone liquido
$(20 \%)$
Clorexidina
$(0 \%)$
Ipoclorito di sodio
$(80 \%)$ Risposta corretta

Domanda 10. Quale tra questi abbinamenti è quello corretto per rimuovere fibrina dal fondo di lesione e contemporaneamente proteggere il bordo di lesione:

Idrogel + iodiopovidone $\quad(30 \%)$

Fibrinolitico + ossido di zinco $(70 \%)$ Risposta corretta

Fibrinolitico + iodiopovidone

in crema $\quad(0 \%)$

Miele + iodiopovidone crema $\quad(0 \%)$ 


\section{DISCUSSIONE}

Dal primo questionario somministrato è emerso che le conoscenze generali sono abbastanza buone ma con ampia possibilità di miglioramento.

Di 10 domande solo a due hanno risposto correttamente tutti gli infermieri. Le altre sono andate abbastanza bene, più del $50 \%$ di loro conosceva la risposta esatta.

Una delle domande che è andata peggio è stata la domanda ${ }^{\circ} 6$ relativa al gold standard del trattamento delle ulcere venose: a questa domanda solo 3 infermieri su 10 hanno risposto correttamente (il 30\%). Gli altri 7 infermieri hanno ritenuto che il trattamento migliore per le ulcere venose fosse il scegliere la medicazione avanzata più idonea. Questo tipo di risposta mi ha fatto pensare a quanto gli infermieri, nella cura della lesione che si trovano davanti, perdano talvolta di vista la persona nella sua totalità e si concentrino maggiormente sulla sua problematica. E' stato chiaro che in questo caso il mio intervento doveva essere incisivo sul fatto che una lesione venosa è da trattare partendo dalla problematica che la genera.

Le risposte alla domanda $n^{\circ} 7$ relativa a ciò che bisogna fare come intervento primario di fronte ad una lesione da pressione, dove la risposta corretta era "lo scarico del peso", non me le aspettavo. Il 100\% ha risposto che l'intervento più importante per una lesione da pressione fosse la medicazione giusta ignorando completamente la risposta corretta. Questo dato è stato discusso durante il lavoro di gruppo in quanto significativo. Il motivo della risposta errata sembrava essere, per la maggior parte degli infermieri, il dare per scontato lo scarico del peso, come se nemmeno questo fosse da considerare intervento di cura.

La domanda sulla detersione, ${ }^{\circ} 1$, che chiedeva quale fosse il prodotto appropriato per effettuarla, ha fatto emergere alcuni dubbi sulla distinzione tra lavaggio e detersione. Il $70 \%$ ha risposto soluzione fisiologica o ringer lattato, il 30\% invece ha risposto acqua e sapone erroneamente. Questo tipo di risultato in questa domanda mi ha fatto sospettare che confondessero i termini lavaggio e detersione. Il fatto di confondere una pratica con l'altra è stato quindi chiarito nel lavoro di gruppo e nello studio individuale, in quanto il materiale che gli ho fornito trattava accuratamente questo capitolo.

La domanda $n^{\circ} 5$ che riguarda il trattamento della pelle sollevata e ancora attaccata in caso di skin tears, ha generato un po' di confusione. Gli infermieri mi hanno detto che effettivamente vecchie pratiche a loro insegnate davano per necessario la rimozione della pelle che si era sollevata durate il trauma dell'anziano, in modo tale da poter fare riformare la pelle sana. Per questo, il $40 \%$ di loro ha dato la risposta sbagliata.

Dal confronto fatto tra i risultati ottenuti nel primo test e in quello finale, il metodo del PBL si è rivelato molto utile in questo gruppo di infermieri, infatti, 8 domande hanno ottenuto il $100 \%$ delle risposte corrette, mentre la $\mathrm{n}^{\circ} 6$ e la $\mathrm{n}^{\circ} 8$ hanno comunque mostrato un miglioramento delle risposte rispetto al test iniziale. Nella domanda $n^{\circ} 6$ sono passati da un $30 \%$ di risposte corrette a un $80 \%$, nella domanda $\mathrm{n}^{\circ} 8$ (relativa alla tecnica meno dolorosa di debridement) le risposte corrette sono passate da un $70 \%$ a un $90 \%$ di risposte corrette.

\section{CONCLUSIONI}

Dai dati emersi nell'applicazione di questa metodologia si possono dedurre diversi aspetti positivi che vanno a supporto della mia tesi.

Innanzitutto, come dato oggettivo, è stato ottenuto un miglioramento delle percentuali di risposte corrette al test finale. Questo fa dedurre quanto il metodo del PBL riesca a fare ottenere risultati positivi fin da subito. Questo dato acquista ancora più valore siccome il miglioramento che si ottiene dal punto di vista delle conoscenze teoriche è immediatamente fruibile nella realtà quotidiana di lavoro.

Se si pensa a quanto sia importante la combinazione tra teoria e pratica nella cura delle lesioni, ecco emergere il pensiero che potere in poco tempo correggere gli errori di uno o dell'altro aspetto sia una grande risorsa a nostra disposizione da potere proporre come alternativa ai corsi in plenaria.

Durante le sedute di PBL ho avuto modo di ascoltare le opinioni dei partecipanti riguardo la metodologia proposta rispetto a quella tradizionale: molti hanno sottolineato quanto fosse stato utile questo tipo di lavoro di gruppo rispetto a quello plenario come prevede la quasi totalità dei corsi di aggiornamento. Mi hanno confidato che spesso, proprio per la modalità con cui venivano esposti, molti contenuti dopo poco tempo non venivano più ricordati, mentre rimanevano solo quelli che poi riuscivano a mettere in pratica nella quotidianità lavorativa.

Una delle note positive che ho potuto osservare è come, durante tutte le ore del lavoro in gruppo, nessuno degli infermieri abbia mai avuto un calo di attenzione, mentre tutti hanno cercato di dare le risposte corrette o esprimere la propria opinione riguardo a ciò che si stava trattando.

Il momento del confronto relativo alla scelta della medicazione, è stato molto pacato nei toni e collaborativo, ognuno ha espresso la propria opinione senza sminuire le idee altrui o le proprie. Il punto di forza di questo tipo di lavoro mi è stato riferito essere proprio la libertà con cui i partecipanti si sono sentiti di intervenire, di fare domande, ma ancora più di tutto di potere sbagliare e sapere che in realtà proprio su ciò che sbagliavano avrebbero poi ottenuto la risposta corretta, mentre, negli altri corsi di aggiornamento, molti non volevano parlare per paura di intervenire in pubblico, di essere giudicati o di fare brutta figura. 
È vero che i dati a supporto di questa tesi non sono statisticamente significativi, ma essendo una proposta di metodologia di insegnamento, spero che i risultati positivi e le opinioni entusiaste dei partecipanti possano invogliare chi si occupa di formazione a prenderlo in considerazione per corsi di aggiornamento futuri. Nonostante io creda che la metodologia del PBL sia un valido strumento di insegnamento, è chiaro che non può essere applicata ad ogni contesto formativo e quindi andare a sostituire totalmente i corsi di aggiornamento in plenaria. Si potrebbe, invece, applicare a quelle realtà in cui lavorano un numero di infermieri limitato, oppure anche ai reparti ospedalieri in modo da potere lavorare in piccoli gruppi senza creare disagi all'organizzazione del lavoro. Si potrebbe procedere, a mio parere, a distribuire questionari per verificare il livello di conoscenza degli infermieri, i punti critici e di forza, e organizzare di conseguenza i corsi basandosi sui dati raccolti. Con questa metodologia si potrebbe, col passare del tempo, uniformare il livello di conoscenza di tutti gli infermieri della stessa unità operativa, diversificare i percorsi di formazione, approfondire ulteriormente certi aspetti. Questa metodologia, inoltre, potrebbe essere utilizzata per la formazione di diverse figure professionali (medici, infermieri, OSS, fisioterapisti, ecc.) perché è adattabile al contesto in cui ci si trova.

Purtroppo nel mondo delle lesioni c'è ancora molta confusione $\mathrm{e}$, da esperienze di reparto, mi è stato descritto come gli infermieri dello stesso contesto lavorativo, davanti alla stessa lesione, abbiano opinioni differenti riguardo la medicazione più appropriata. Se riuscissimo a uniformare la conoscenza riguardo le medicazioni e le lesioni nei reparti o nelle strutture e fare intervenire un esperto in Wound Care quando necessario, ci potrebbero essere miglioramenti significativi anche riguardo ai numeri spaventosi della frequenza dell'insorgenza delle lesioni e un grande miglioramento nella loro cura e guarigione.

\section{BIBLIOGRAFIA}

1. Knowles M. The modern practice of adult education. Andragogy versus pedagogy. Chicago: Association Press/Follett Publishing; 1970.

2. Cox CW, Gunderman RB. Andragogic approaches to continuing medical education. Acad Radiol 2017;24:1325-6.

3. D'Ottavio AE. Theoretical and behavioral features to be considered when planning and implementing a pbl curriculum. Rev Fac Cien Med Univ Nac Cordoba 2016;73:311-3.

4. Schmidt HG. Problem-based learning: rationale and description. Med Educ 1983;17:11-16.

5. Lotti A. Il tutor facilitatore dell'apprendimento di competenze intellettive. In Sasso L, Lotti A, Gamberoni L, eds. Il tutor per le professioni sanitarie. Roma: Carocci; 2005.

6. Petrella F, Abbritti F, Bachetti F, et al. Progetto S.I.U.C. (Studio italiano ulcere cutanee) (1 gennaio 2015-31 dicembre 2016). Dati conclusivi. Ital J Wound Care 2017;1:1-8. 